Reviews and Meta-Analysis

\title{
Measuring the health of populations: explaining composite indicators
}

\author{
Adnan A. Hyder, Prasanthi Puvanachandra, Richard H. Morrow \\ International Injury Research Unit, Department of International Health, Johns Hopkins Bloomberg \\ School of Public Health, Baltimore, Maryland, USA
}

\begin{abstract}
Significance for public health
Health programmes are evaluated for impact on the burden of mortality, morbidity and disability in populations. Increasingly, summary measures of population health, such as healthy life years (HeaLYs), disability-adjusted life years (DALYs), and quality-adjusted life years (QALYs), are being used to assess the impact and effectiveness of health interventions, and are useful tools for guiding resource allocation and planning efficient (and equitable) health care systems. A thorough understanding of these measures is crucial for public health professionals.
\end{abstract}

\begin{abstract}
Indicators that summarise the health status of a population and that provide comparable measures of a population disease burden are increasingly vital tools for health policy decision making. Decisions concerning health systems across the world are greatly affected by changes in disease profiles and population dynamics, and must develop the capacity to respond to such changes effectively within the resources of each nation. Decisions must be based on evidence of the patterns of diseases, their risk factors and the effectiveness of alternative interventions. This paper focuses on the main approaches used for developing summary measures that include mortality and morbidity occurring in a population. It discusses the rationale for composite measures and reviews the origins of each main approach. The paper also examines methodological differences among these approaches making explicit the value choices that each entails, outlines the advantages and limitations of each measure, and shows how they relate to one another.
\end{abstract}

\section{Introduction}

Recent developments in the measurement of population health status and disease burden include the increasing use of summary composite measures of health that combine the mortality and morbidity effects of diseases into a single indicator. Measures of health status that combine mortality and morbidity facilitate comparisons within and across populations. They are used to estimate the quantitative health benefits from interventions and serve as tools to assist in the allocation of resources. The construction of such measures entails two major processes: the measurement of healthy life, including losses of time from mortality and disability; and valuing life, which incorporates issues of duration, age, extent of future life, productivity, dependency, and equity. ${ }^{1}$

This paper focuses on the main approaches used for developing composite measures of population health status that summarise mortality and morbidity occurring in a population through the use of a single number. It discusses the rationale for composite measures, reviews the origins of selected main approaches, examines methodological differences among these approaches, makes explicit the value choices that each entails, outlines the advantages and limitations of each, and shows how they relate to one another.

\section{Summary measures}

Two types of composite summary measures have been developed: health gap measures such as healthy life years (HeaLYs), disabilityadjusted life years (DALYs), and quality-adjusted life years (QALYs), and health expectancies such as disability-free life expectancy (DFLE) or health-adjusted life expectancy (HALE). Both types use healthy life time lost through disability and death as a common measure of the impact of mortality and non-fatal health outcomes. These two types of measures are complementary and have been discussed by Murray and Lopez. ${ }^{2,3}$ HeaLYs, DALYs, and QALYs are developed on the basis of disability and death attributable to a specific disease in an individual person. In their construction, great care is taken that there is categorical attribution using the International Classification of Disease (ICD) so that each event (death or disability) is mutually exclusive and collectively exhaustive. With these measures, therefore, summing deaths and disabilities from each disease provides the total amount of death and disability for the population (a property termed additive decomposition). Health gap measures have this property whereas health expectancies do not.

A number of composite summary indicators for burden of disease assessment have been developed. We shall review four families. Three of these are of the health gap type: the healthy life year (HeaLY), the disability-adjusted life year (DALY), and the quality-adjusted life year (QALY), and one is of the health expectancy type, namely the healthadjusted life expectancy (HALE). These metrics are the most commonly used and are, therefore, discussed below.

\section{Healthy life year}

We discuss the healthy life year first because it is conceptually straightforward, serves as a prototype for other health gap indicators, and was the first of the composite measures to be used as a tool in national health planning. ${ }^{4}$ In the early 1980 s the Ghana Health Assessment Team undertook a study to assess the burden of disease in low- and middle-income countries (LMICs). The healthy life year (HeaLY) approach is a direct derivative of that work in Ghana. ${ }^{5}$ The 
HeaLY combines the amount of healthy life lost due to death with that lost due to morbidity. Life lost from a disease due to death is based on the years of life expectancy had the disease not occurred. The information needed to estimate this, in addition to the incidence rate and case fatality ratio, is the age of disease onset, the age of death, and the expectation of life at these ages. Life lost from disability must have comparable dimensions to that lost due to death. The HeaLY includes three components for disability: case disability ratio (CDR, analogous to the case fatality ratio), extent of disability, and duration of disability. The CDR and duration of disability can be determined objectively, but assessment of the extent of disability, which ranges from 0 (no disability) to 1 (equivalent to death) may have a substantial subjective element. ${ }^{1}$

The healthy life approach focuses on the pathogenesis and natural history of disease as the conceptual framework for assessing morbidity and mortality, and for interpreting the effects of various interventions. Interventions may also be directed at reducing identifiable risk factors, such as tobacco smoking or risky sexual behaviour. To the extent that risk reduction can be translated into disease reduction, the approach to measuring the benefits and costs of a risk reduction intervention programme remains the same as that for disease reduction.

For the purpose of estimating healthy life lost from disease or gained from interventions, disease is defined according to the $10^{\text {th }}$ revision of the International Statistical Classification of Diseases and Related Health Problems (known as the ICD-10), a medical classification list for the coding of diseases, signs and symptoms, abnormal findings, complaints, social circumstances, and external causes of injury or diseases. $^{6}$ The definition of variables and formula to calculate HeaLYs are summarised in Table 1.

The expectation of life in HeaLYs, as with DALYs, is based on expectations of what should occur under optimal circumstances. For example, the life expectancy for women in Japan based on the West model life tables (The West model life table gives female life expectancy for women in Japan as 82.5 years).,8 The CDR for most diseases is 1 because all cases labelled as a disease in the ICD are, therefore, disabled to varying degrees and duration. The duration of disability can be either temporary or permanent (lifelong). If the disability is temporary, then $D_{t}$ is the duration of that disability until recovery (Table 1 ). If the disability is permanent and the disease does not affect life expectation, then the expectation of life at age of onset of disease can be used. On the other hand, if the disability is permanent and the disease does reduce life expectancy, then the expectation of life at age of onset reduced by the difference between ages of fatality and onset can be used. A disability severity scale needs to be used to estimate extent (severity) of the disability. This review does not attempt to provide a critique of the methods and scales used in assessing the extent of disability indicator used in QALYs or DALYs but rather focuses on the basic constructs that are involved in each of these approaches outlining how they relate to one another.

The healthy life lost from death and from disability are added and expressed as the total years of life lost per 1000 population per year; the loss is attributed to the year in which disease onset occurs and includes the stream of life lost from disability and death at any time after onset, even if these events happen many years later. This is a prospective view of the event (disease onset) and its natural history (or as modified by interventions) over time.

An important benefit of the HeaLY formulation is that the effects of different kinds of interventions can be readily explored to determine their expected gains in healthy life. Interventions may usefully be divided into two broad categories: those that are used to prevent the initiation of the disease process, and those that are used to treat a disease process already under way, which includes rehabilitative care. Some interventions fall into both categories. The primary effect of preventive strategies is to reduce the incidence of new cases of disease. The main effects of treatment strategies are to interfere with the natural history of the disease process, thereby reducing the case fatality and/or case disability ratios or extending life by providing a later age at death for conditions such as diabetes and AIDS (for an example of the application of the HeaLY measure see Hyder et al. ${ }^{5}$

\section{Disability-adjusted life year}

The disability-adjusted life year (DALY) is a health gap population summary measure that combines time lost due to disability with that which is due to death (life that would have been expected had the disease not occurred) in a manner similar to the healthy life year measure. It first appeared in the World Development Report of $1993^{9}$ and has become the most widely used composite measure of population health. ${ }^{2,3,10-13}$ DALYs are calculated as two separate components for the measurement of life lost due to disease, and they also directly include three social value choices. The two components are: i) years of life lost (YLL), the loss of healthy life from death; and ii) years of life lived with disability (YLD), the loss of healthy life from disability. The social value choices that can be included are: i) life expectation values; ii) discount

Table 1. Variables for estimating healthy life years (HeaLYs).

\begin{tabular}{|c|c|c|}
\hline Description & Unit & Symbol \\
\hline Incidence rate per 1000 population per year & per 1000 per year & I \\
\hline Average age at onset & years & Ao \\
\hline Average age at death & years & $A_{f}$ \\
\hline Expectation of life at age of onset & years & $\mathrm{E}(\mathrm{Ao})$ \\
\hline Expectation of life at age of death & years & $\mathrm{E}\left(\mathrm{A}_{\mathrm{f}}\right)$ \\
\hline Case fatality ratio: proportion of those developing the disease who die from the disease & $0.00-1.00$ & CFR \\
\hline Case disability ratio: proportion of those developing the disease who have disability from the disease & $0.00-1.00$ & CDR \\
\hline Extent of disability (from none to complete disability equivalent to death) & $0.00-1.00$ & De \\
\hline Duration of disability in years & years & $\mathrm{D}_{\mathrm{t}}^{\circ}$ \\
\hline Healthy life years lost per 1000 population per year & HeaLYs per 1000 per year & HeaLYs \\
\hline \multicolumn{3}{|l|}{ HeaLY $=I^{*}\left\{\left[C F R *\left\{E\left(A_{0}\right)-\left[A_{f}-A_{0}\right]\right\}\right]+\left[C D R * D_{e} * D_{t}\right]\right\}$} \\
\hline
\end{tabular}


rates for future life; and iii) weighting for life lived at different ages, as discussed below. The calculation for YLL in a population uses the age distribution of all deaths by cause in one year multiplied by life expectation at each age to estimate loss of life for each disease that would have been expected if that disease had not occurred. The expectation of life is obtained from a model life table based on best achievable low levels of mortality, such as in Japan, ${ }^{8}$ and thus the DALY (and the HeaLY) directly incorporates this social value choice.

For disability, the DALY uses estimates of incidence, duration, and severity to calculate the time lived with disability (YLD) for each disease. The YLD component equals the number of incident cases in the period multiplied by the average duration of disease multiplied by a weight factor for the degree of severity (extent) of disability from the disease. The second social value choice directly incorporated in the DALY is the discount rate of $3 \%$ per annum. This social time preference has been used for most estimates; however, DALY results discounted at $0 \%$ are also available.

The third social value choice concerns weighting life lived at different ages. DALYs are age-weighted according to an arbitrary exponential curve designed to give the most value to life lived as a young adult.5,9 Weighting by age was the most controversial component of the DALY when they appeared and caused great dissent among other health professionals (see below Valuing Life Lived at Different Ages). ${ }^{2}$ Recent DALY listings of Global Burden of Disease (GBD) studies also include results with no age weighting (all years equally valued). ${ }^{14}$ It has been argued that age weighting of DALYs does not affect final results, but this depends on the purpose for making the estimates and has been challenged. ${ }^{5,15-17}$ The definition of variables and formula to calculate DALYs are summarised in Table 2.

Note that an important difference between the HeaLY and DALY is that the starting point for the HeaLY is the onset of disease; the loss of healthy life is based on the natural history of the disease (as modified by interventions). This is true for the YLD component of the DALY, but the YLL is based on mortality in the current year. In a steady state there is no difference, but when there is a changing incidence, such as with HIV in many parts of the globe, the DALY approach can greatly understate the true situation. ${ }^{18}$

The calculation for DALYs can be expressed in the form of an integral that was first published in the World Bank literature. ${ }^{2}$ This single equation incorporating all technical and value choices has the advantage of standardisation to ensure comparability of the multiple calcula- tions undertaken in the Global Burden of Disease studies and, certainly, it has greatly facilitated the actual computations. However, for national and local priority setting, it may be preferable to use an indicator constructed such that the social value choices can be adjusted to suit national and local preferences. ${ }^{1,5,19}$ Recent DALY formulations allow for this; as a result, it is useful to think of DALYs as a family of related measures. ${ }^{13}$

HeaLYs and DALYs are both health gap measures and can be considered in the same family of measures. In fact, DALYs exactly equal HeaLYs when: i) the condition in question is in a steady state or equilibrium (that is the Incidence, CFR, and Disability variables remain constant during the time intervals under consideration); ii) age weighting is not applied $(\mathrm{K}=0)$; and iii) the same measures of disability (weights) are used (Table 2).

\section{Quality-adjusted life year}

The quality-adjusted life year (QALY) was introduced in 1976 to provide a guiding principle for selecting among alternative tertiary health care interventions..$^{20}$ The idea was to develop a single measure of quality of life in order to compare expected outcomes for a disease from different interventions; a measure that valued possible health states for their quality of life as well as their duration.

The central notion behind the QALY is that a year of life spent in one health state may be preferred to a year spent in another. It is a generic measure that sums time spent in different health states using weights for each health state on a scale of 0.00 (dead) to 1.00 (perfectly healthy) i.e. the arithmetic product of duration of life and a measure of quality of life (health state weight) (Table 2). So, five years of perfect health $=5$ QALYs while two years in a state measured as 0.5 of perfect health followed by three years of perfect health $=4$ QALYs.

The QALY was originally developed as a differentiating indicator for individual choices among tertiary health care procedures, not as a measure of disease burden in a population. It was used to assess individual preferences for different health outcomes from alternative interventions. ${ }^{1}$ Since its introduction, a wide variety of QALY measures have been developed, along with an extensive literature on alternative methods incorporating a range of disability domains and a diversity of methods to assign weights to generate QALYs. ${ }^{21,22}$ The most widely used measure is the EQ-5D (European Quality of Life with Five Domains and

Table 2. Variables and formula for Disability Adjusted Life Years (DALYs) and basic formulation for Quality-Adjusted Life Years (QALYs).

\begin{tabular}{|c|c|c|}
\hline Description & Unit & Symbol \\
\hline Years of life lost due to death before expectation of life had the disease not occurred & years & YLL \\
\hline Years lost due to disability multiplied by disability weight & years & YLD \\
\hline Number of deaths & number & $\mathrm{N}$ \\
\hline Number of incident cases & number & I \\
\hline Life expectation or duration of disability & years & DW \\
\hline Discount rate & $\%$ & r \\
\hline Age-weighting correction constant & 0.1658 & $\mathrm{C}$ \\
\hline Parameter for age-weighting function & 0.04 & b \\
\hline Age at onset of disease & years & $\mathrm{a}$ \\
\hline Parameter for setting age-weighting & 1 or 0 & K \\
\hline \multicolumn{3}{|c|}{$\begin{array}{l}D A L Y=Y L L+Y L D \text { where } Y L L=N C e^{(r a)} /(+r)^{2}\left[e^{(+r)(L+a)}[-(+r)(L+a)-1]-e^{(+r) a}[-(r) a-1]\right] \\
Y L D=I D W\left\{K C e^{(r a)} /(+r)^{2}\left[e^{(+r)(L+a)}[-(+r)(L+a)-1]-e^{(+r) a}[-(r) a-1]\right]+(1-K)(L / r)\left(1-e^{r L}\right)\right\} \\
\text { QALYs (basic formulation) QALY= Life years lived (duration) x quality of life (health state) indicator }\end{array}$} \\
\hline
\end{tabular}

${ }^{\circ}$ These would be based on a number of different quality of life scales., ${ }^{2,3}$ 
3 levels of quality for each Domain). ${ }^{23}$

Perhaps the most important use of QALYs has been as a common denominator to measure utility in cost-utility analysis (CUA) and effectiveness in cost-effectiveness analysis (CEA) to assist in resource allocation among alternative health interventions by ranking interventions in terms of cost per QALY. ${ }^{21,24}$ An early and widely publicised attempt to make the best use of health care resources by maximising QALYs per dollar spent was the well-intentioned but rather unfortunate effort in Oregon in the early 1990s. ${ }^{25,26}$ In the UK, as part of its 1997 NHS reforms, the National Institute for Clinical Excellence (NICE) was created to advise about the effectiveness and cost effectiveness of various health interventions. In order to introduce economic considerations in addition to medical judgments for the allocation of resources, NICE has produced a large collection of studies on the cost per QALY produced by the interventions it appraises (www.nice.org.uk).

The QALY as originally used is essentially equivalent to the YLD of the DALY. In fact, it would be similar to the YLD when: $\mathrm{i})$ there is no discounting $(\mathrm{r}=0)$; ii) no age weighting is used $(\mathrm{K}=0)$; and iii) the same disability weights are used. More recently (as used in some cost-effectiveness studies) QALYs have also incorporated life expectation.

\section{The health-adjusted life expectancies}

There are several types of health expectancies in the literature. During the 1990s, disability free life expectancy (DFLE) and related measures were calculated for many countries. ${ }^{27,28}$ However, these measures incorporate a dichotomous weighting scheme in which time spent in any health state categorised as disabled is assigned, arbitrarily, a weight of zero (equivalent to death). Thus, DFLE is not sensitive to differences in the severity distribution of disability in populations. In contrast, the disability-adjusted life expectancy (DALE) adds up expectation of life for different health states with adjustment for severity weights.

The term DALEs was replaced by the term HALEs (health-adjusted life expectancy) by the World Health Organization (WHO) in 2001 and this is the term that we will use here. The HALE is a composite summary measure of population health status that belongs to the family of health expectancies. It summarises the expected number of years to be lived in what might be termed the equivalent of full health. Some consider the HALE to provide the best available summary measure for measuring the overall level of health for populations. ${ }^{27}$ The WHO has used it as a measure of the average level of health of the populations of member states for annual reporting.

Health expectancy indices combine the mortality experience of a population with the disability experience. Some versions of the HALE are calculated using the prevalence of disability at each age in order to divide the years of life expected at each age according to a life table cohort into years with and without disability. Mortality is captured by using a life table method, while the disability component is expressed by additions of prevalence of various disabilities within the life table. This indicator allows an assessment of the proportion of life spent in disabled states. When compared with the total expectation of life, this translates to a measure of the total disability burden in a population. The various methods and specific indicators have been compared in the literature. ${ }^{28}$ Alternative methods are given in the WHO National Burden of Disease Studies manual. ${ }^{27}$

As originally designed, the HALE does not relate to specific diseases but rather to the average extent of disability among that proportion in each age group that is disabled. The lack of correlation between a condition or disease entity and the measure makes it intuitively less valuable for resource allocation and cost-effectiveness calculations.

\section{Valuing life: social issues}

To construct composite measures of population health, important social value choices must be made. Choices about what expectation for life should be used and about valuing life lived at different ages, valuing future life as compared with the present, valuing life in terms of economic and social productivity, and valuing equity in relation to efficiency all raise major ethical concerns.

\section{Expectation of life}

Years of life lost due to death and to chronic disability are based on life expected had the disease not occurred. To estimate the expectation of life in a population, a choice must be made as to whether to use a local, national or a model life table. This choice should be determined by the purpose of the study. For assisting in national and local decision making, it may be more suitable to use national life tables based on the mortality and fertility of the population in question than on model life tables. On the other hand, a model life table can be selected to reflect the best health state possible in the world, such as the Coale and Guo West model. ${ }^{8}$ This selection allows a fair comparison with other countries. For example, from a global perspective, it would be unfair to use national life tables to compare gains that could be achieved from a particular intervention in Ghana with those in the United Kingdom, even if both costs and lives saved were the same in each country. The reason is that those lives saved in Ghana would have a lower life expectancy than those in the United Kingdom, resulting in less healthy life saved for the same expenditure. From the global viewpoint, such use would erroneously prioritise the action to fund the intervention in the United Kingdom because it would produce more healthy life per expenditure than it would in Ghana.

Model life tables in common use are the United Nations model life tables and the Coale and Demeney life tables which were used in the HeaLY and GBD studies. ${ }^{2,5,8}$ The West model life table does not refer to any geographical entity but is considered to represent a mortality pattern typical of the most technologically advanced countries. Level 26 has a female life expectancy at birth of 82.5 years, as actually experienced by women in Japan; therefore, it represents a level that could be achievable elsewhere.

\section{Valuing life lived at different ages}

Age weighting refers to the valuing of a year of life according to the age at which it is lived. This immediately raises questions as to the basis for valuing human life. Is a day of anyone's life of the same value as that of anyone else? Does the value vary with age, economic productivity, or social status? Should life itself be valued separately from what is done with that life?

The Ghana Health Assessment Team judged that all human life was intrinsically valuable and that a given duration of any life was equal in value to that of any other life. ${ }^{4}$ The valuing of a year of life equally, irrespective of age, has been considered egalitarian.,29 This choice remained in the development of the HeaLY approach: a year of life lived at any age is equally valued.

The original DALY formulation assigned a quasi-exponential function to provide a value chosen so that life lived as a dependent (e.g., infants, children, and the elderly) is given less value than life lived during the productive years. In this decision, the intrinsic value of life increases from 0 at birth to a maximum at 25 years of age and declines thereafter, so that a day of life of a 50 -year old is worth about $25 \%$ less than that of a 25 -year old. Paradoxically, the age weighting used in the original DALY formulation leads to higher valuation of life lived at under 15 years of age than does the HeaLY formulation, in which life lived at all ages is valued equally. ${ }^{5,16}$ Current formulations of the DALY 
leave age weighting as an option, and such weighting is not used with the HALE. If it is decided that healthy life should be valued according to economic and social productivity, then an alternative to age weighting might be to explicitly add a productivity factor or subtract for the societal costs of dependents, such as education (see below Valuing life for its economic productivity).

\section{Discounting}

Discounting is the process for determining the present value of future events. Social time preference takes into account the phenomenon that people value events at present more highly than those in the future (independent of inflation and of uncertainty). For investments in other sectors, time preference is normally taken into account by discounting future returns and costs by some appropriate discount rate. It can be considered the inverse of an interest rate. The main issue concerning discounting in relation to summary measures is whether discounting life itself is appropriate, though discounting the future value of what is produced by healthy life appears to be less contentious. ${ }^{1}$

Discounting has been applied in the health sector because both the losses from a disease and the benefits from a health intervention often occur in the future. ${ }^{30} \mathrm{An}$ intervention today may not produce immediate benefits (such as in immunisation), or it may result in benefits being sustained over a long time (such as in supplementary nutrition). The costs for these interventions must be borne now, but benefits take place in the future and are less valued than if they could occur now. This is equivalent to investing money now in order to obtain more in the future. Because of social time preference, a healthy life year now has greater intrinsic value to an individual or community than one in the future. ${ }^{31,32}$

There is no consensus on the choice of a discount rate in health, but most agree that it should be lower than that in the private commercial sector. The World Development Report (WDR) in 1993 and the GBD studies discounted at 3\% a year; in lieu of other information, this rate has come to be used in most international health cost-effectiveness studies. ${ }^{9}$ However, the impact of using a range of different discount rates, including zero, should be explored with each study.

\section{Valuing life for its economic productivity}

In general, productivity may be attributed to adults aged 15-64 years of age, and individuals in these age groups could be given a higher value for their time spent at these ages. Those under 15 years of age and over 65 may be considered as dependents and given a lower value. There are many variations for differential valuing, including type of employment. People at different socio-economic levels in a society are expected to have different capacities for productivity, yet to value life according to income levels or social class would not seem fair, and would not generally be acceptable. In poor countries, the value of marginal wages for subsistence agriculture is negligible, but the value of the workers' lives certainly is not.

A fundamental question is whether to consider adding a productivity component to the summary measure. Health issues do not readily conform to the requirements of market economics; information is inadequate, and misinformation is rife on the part of the providers as well as the public. Externalities from good health are generally large. Demand for costly services is largely determined by the health care providers rather than by the consumers. Competitive market forces have not worked well for those in greatest need. In the private sector, demand for services is clearly related to productivity and willingness (and ability) to pay. If left to market forces alone, inequitable distribution of healthcare would be inevitable.

\section{Valuing equity in relation to efficiency}

Decisions based on cost-effectiveness (e.g., cost per healthy life year) may not accord well with concerns about equity. CEA calculations are generally indifferent to equity; they are designed to steer interventions to what is efficient, whatever the differential need may be. To meet the requirements of equity, health system planners need to go beyond ensuring equality of access to health care and must require a balance so that health system responses are in accord with equity as well as efficiency.

Provided that composite indicator information is available according to socio-economic and vulnerable groups, use of these summary indicators as tools for equity by calculating healthy life per dollar to be gained by all socio-economic and vulnerable groups could readily be undertaken. It would be straightforward to assess the impact of specific health decisions to ensure that they enhance equity. Summary measures such as HeaLYs and DALYs can be used to guide allocation of resources to ensure equitable distribution of those resources so as to reach those most in need. Cost-effectiveness by itself does not provide adequate guidance; equity should be an additional criterion to govern the distribution of societal benefits.

\section{Discussion}

The health of populations is the fundamental concern of global public health. The first step in the pursuit of population health improvement is the measurement of health and disease. Measurement is required to establish the magnitude of disease problems, define causal factors, explore potential solutions, and determine the impact of interventions. Measuring the impact of diseases on populations in terms of mortality and morbidity and their consequences is essential for planning effective ways to reduce the burden of illness and for setting priorities.

The burden of disease in populations has been gauged in many ways. Examples include measures of mortality such as infant mortality rates, demographic measures such as expectation of life at birth, and measures of morbidity such as days away from work. However, for purposes of comparison among populations, and for assisting in health planning and resource allocation, a common denominator combining these factors is needed. Summary measures of population health based upon the amount of healthy life time lost from disability and from death have been developed to serve that purpose.

Composite indicators (such as HeaLYs, DALYs and QALYs) use duration of time (years, weeks, days) to measure the loss of healthy life from disease and the gain from interventions. These are increasingly recognised as being important tools for assisting health related decision making. However, in order to avoid misuse, it is critical for those using them to understand the underlying assumptions and limitations, and also to meet the rather formidable data requirements. These summary measures could be used to examine the burden of disease amongst sub-populations, according to socio-cultural-economic category and especially to those groups most vulnerable, and used for ensuring that health-related decisions consider equity as well as cost-effective criteria.

Having said that, it is important to mention that while these composite measures take ICD diagnoses as a starting point for mortality and disability, the appropriateness of the latter has been criticized..$^{33}$ The UN Convention on the Rights of Persons with Disabilities defines disability as an evolving concept resulting from the interaction of impairment (when the disease process is already underway) and physical and attitudinal environments. ${ }^{34}$ In this way, disability cannot be disentangled from the social context in which these interactions take place, and this understanding is not necessarily reflected in the current measures. New approaches for measuring disability in the general population based on the WHO's International Classification of Functioning, 
Disability and Health (ICF) have been developed and may be complementary to ICD. ${ }^{35}$

Trends in disease burden provide important clues to the success of ongoing health programmes and the need for development of new interventions. At the same time, they reflect non-health factors that are important to the production or maintenance of health in populations. Inter-country and inter-regional comparisons allow for measuring progress among nations. They can highlight inequalities in health status and examine these in relation to social, economic, educational, and other factors as well.

Health systems across the world are greatly affected by changes in disease profiles and population dynamics. These systems must develop the capacity to respond to such changes effectively within the resources of each nation. Decisions should be based on evidence about the patterns of diseases, their risk factors and effectiveness of alternative interventions. Timely collection and analysis of appropriate, highquality data to support such evidence are a prerequisite for improving equitable global health development.

Correspondence: Dr. Adnan A. Hyder, International Injury Research Unit, Department of International Health, Johns Hopkins Bloomberg School of Public Health, 615 North Wolfe Street, Suite E-8132, Baltimore, MD 21205, USA.

Tel. +001.410.955.3928 - Fax: +001.410.614.1419.

E-mail: ahyder@jhsph.edu

Key words: composite indicators, burden of disease, summary measures, epidemiology, health systems.

Contributions: AAH, study concept, prepared first draft, revised the manuscript; PP, RHM manuscript revision. All authors gave their final approval for the version to be published.

Conflict of interests: the authors declare no conflict of interests.

Acknowledgments: the authors would like to acknowledge the valuable editorial contributions of Jeffrey C. Lunnen.

Received for publication: 14 June 2012.

Accepted for publication: 13 November 2012.

(C) Copyright A.A. Hyder et al., 2012

Licensee PAGEPress, Italy

Journal of Public Health Research 2012; 1:e35

doi:10.4081/jphr.2012.e35

This work is licensed under a Creative Commons Attribution NonCommercial 3.0 License (CC BY-NC 3.0).

\section{References}

1. Morrow RH, Bryant JH. Health policy approaches to measuring and valuing human life: Conceptual and ethical issues. Am J Public Health 1995;85:1345-6.

2. Murray CJL, Lopez AD. The Global Burden of Disease 1990. Geneva: World Health Organization; 1996.

3. Murray CJL, Lopez AD. Global health statistics 1990. Geneva: World Health Organization; 1996

4. Ghana Health Assessment Team. A quantitative method for assessing the health impact of different diseases in less developed countries. Int J Epidemiol 1981;10:73-80.

5. Hyder AA, Rotllant G, Morrow RH. Measuring the burden of disease: Healthy life years. Am J Public Health 1998;88:196-202.

6. WHO. The International Statistical Classification of Diseases and Related Health Problems. 10th Revision. Available from: http:/apps.who.int/classifications/icd10/browse/2010/en.
7. Coale AJ, Demeney P. Regional model life tables and stable populations. New York: Academic Press; 1983.

8. Coale AJ, Guo G. Revised regional model life tables at very low levels of mortality. Popul Index 1989;55:613-643.

9. World Bank. World development report 1993: investing in health. New York: Oxford University Press; 1993.

10. Murray CLJ, Lopez AD. On the comparable quantification of health risks: lessons from the Global Burden of Disease Study. Epidemiology 1999;10:594-605.

11. Murray CJL, Salomon J, Mathers C, Lopez AD. Summary measures of population health: concepts, ethics, measurement and applications. Geneva: World Health Organization; 2002.

12. Lopez AD, Ahmad 0, Guillot M, et al. World Mortality in 2000: Life Tables for 191 Countries. Geneva: World Health Organization; 2002.

13. Jamison DT, Brenan JG, Measham AR, et al. Disease Control Priorities in Developing Countries. New York: Oxford University Press; 2006

14. WHO. The World Health Report 2000. Geneva: World Health Organization; 2000.

15. Anand S, Ranaan-Eliya. Disability adjusted life years: a critical review. Working Paper No. 95-06. Harvard Center for Population and Development Studies; 1996.

16. Barendregt JJ, Bonneux L, Van Der Maas PJ. DALYs: the age weights on balance. Bull World Health Org 1996;74:439-443.

17. Barker C, Green A. Opening the debate on DALYs (disability-adjusted life years). Health Policy Plan 1996;1;179-183.

18. Hyder AA, Morrow RH. Steady state assumptions in DALYs: effect on estimates of HIV impact. J Epidemiol Community Health 1999;53:43-5

19. Bobadilla, JL. Searching for essential health services in low and middle income countries. Washington, DC: Inter American Development Bank; 1998.

20. Zeckhauser R, Shephard D. Where now for saving lives? Law and Contemporary Problems 1976;40:5-45.

21. Kaplan RM. The General Health Policy Model: an integrated approach. In: Spilker B, editor. Quality of life assessment in clinical trials. New York: Raven Press; 1990.

22. Nord E. Unjustified use of the quality of well being scale in priority setting in Oregon. Health Policy 1993;24:45-53.

23. EuroQol Group.. What is EQ-5D? 2010. Available from: http://www.euroqol.org/eq-5d/what-is-eq-5d.html

24. Nord E. Methods for quality adjustment of life years. Soc Sci Med 1992;34:559-69.

25. Adler MD. QALYs and policy evaluation: a new perspective. Yale J Health Policy Law Ethics 2006;6:1-92.

26. Kaplan RM. Utility assessment for estimating quality-adjusted life years. In: FA Sloan (ed.) Valuing health care: costs, benefits, and effectiveness of pharmaceuticals and other medical technologies. Cambridge: Cambridge University Press; 1996.

27. Mathers CD, Vos T, Lopez AD, et al. National Burden of Disease Studies: A Practical Guide. 2nd ed. Global Program on Evidence for Health Policy. Geneva: World Health Organization; 2001.

28. Robine JM. Disability free life expectancy trends in France: 19811991: International comparisons. In: C. Mathers et al. (eds) Advances in health expectancies. Canberra: Australian Institute of Health and Welfare; 1994.

29. Busschbach JJV, Hessing DJ, de Charro FT. The utility of health at different stages of life: A qualitative approach. Soc Sci Med 1993;37:153-8.

30. Sheldon, TA. Discounting in health care decision-making: time for change? J Public Health Med 1992 Sept;14(3):250-6.

31. Gold MR, Siegel JE, Russel LB, Weinstein MC, editors. CostEffectiveness in Health and Medicine. New York: Oxford University 
Press; 1996.

32. Weinstein MC, Siegel JE, Gold MR, Kamlet MS, Russell LB. Recommendations of the Panel on Cost-effectiveness in Health and Medicine. JAMA 1996;276:1253-8.

33. Mont D. Measuring health and disability. Lancet 2007;369:1658-63.

34. United Nations. General Assembly. Final report of the Ad Hoc Committee on a Comprehensive and Integral International Convention on the Protection and Promotion of the Rights and
Dignity of Persons with Disabilities. New York: United Nations; 2006. Available from: http://daccess-dds-ny.un.org/doc/UNDOC/ LTD/N06/645/30/PDF/N0664530.pdf?OpenElement.

35. Madans JH, Loeb ME, Altman BM. Measuring disability and monitoring the UN Convention on the Rights of Persons with Disabilities: the work of the Washington Group on Disability Statistics. BMC Public Health 2011;11:S4. 